\title{
Obstructive sleep apnoea is associated with impaired pictorial memory task acquisition and retention in children
}

\author{
L. Kheirandish-Gozal*, M.R. de Jong*,\# , K. Spruyt*, S.A.J. Chamuleau\# and D. Gozal*
}

ABSTRACT: The aim of our study was to evaluate whether obstructive sleep apnoea (OSA) is associated with impaired acquisition and recall of a pictorial-based memory tasks in children.

54 children with OSA and 17 controls matched for age, sex and ethnicity underwent a sleep study (overnight polysomnogram). Before the sleep study subjects completed a 15-min pictorial memory task acquisition consisting of four trials, followed by a free-recall period to assess retention after $10 \mathrm{~min}$ and the following morning upon awakening.

Children with OSA had a higher obstructive apnoea/hypopnoea index $\left(6.3 \pm 1.5\right.$ events $\cdot h^{-1}$ TST) than controls $\left(0.6 \pm 0.1\right.$ events $\cdot h^{-1}$ TST) $(p<0.0001)$. Mean learning scores in controls over the four consecutive trials were incrementally better than in children with OSA for the four-trial set $(p<0.0001)$. Both immediate $(p<0.0001)$ and overnight recall performances were worse among OSA children $(p<0.0001)$, who also exhibited declines in recall performance that was absent in controls $(p<0.001)$.

Differences in pictorial task acquisition trajectories suggest that children with OSA require more time and an increased number of learning opportunities to reach immediate and long-term recall performances that are reduced compared with controls. Thus, both acquisition and retention of newly learned material are compromised. These findings confirm and expand on the presence of known cognitive deficits in children with OSA.

KEYWORDS: Children, long-term memory, memory consolidation, obstructive sleep apnoea, sleep-disordered breathing

$\mathrm{t}$ is now well-established that sleep in normal children exerts profound changes in respiratory patterns and, therefore, predisposes susceptible individuals to develop respiratory abnormalities, which are collectively referred to as sleep-disordered breathing (SDB) [1, 2]. Paediatric obstructive sleep apnoea (OSA) is the most severe form of SDB [3] and has a prevalence of $1-3 \%$ in otherwise healthy children [4]. OSA has been defined by the American Thoracic Society as a "disorder of breathing during sleep characterized by prolonged partial upper airway obstruction and/or intermittent complete obstruction (obstructive apnoea) that disrupts normal ventilation during sleep and normal sleep patterns" [1]. The most important risk factors for the development of paediatric SDB include adenotonsillar hypertrophy, obesity, craniofacial anomalies and neuromuscular disorders [5-8]. Even though children may outgrow this condition, long-term detrimental effects on several end-organ systems have been recognised and have prompted the need for early detection and intervention $[9,10]$.
Current theory posits that both the sleep fragmentation, which develops as a consequence of repeated arousals, and the intermittent blood gas abnormalities (hypoxia and hypercarbia) that characterise OSA [10-12] may jointly lead to a wide array of morbid consequences. The latter include reduced intelligence and memory, behavioural deficits including attention deficit hyperactivitylike disease, aggressiveness and poor impulse control, as well as failure to thrive, enuresis and cardiovascular dysfunction [10, 13-25]. Conversely, adequate treatment of OSA improves or reverses these morbidities, and is further associated with improved overall quality of life [26] and reduced healthcare costs [27].

In adult subjects, preservation of both rapid eye movement (REM) sleep and non-REM sleep integrity is of great importance to the consolidation of both declarative (factual recall) and nondeclarative memory (procedural skills) [28]. Therefore, disruption of these sleep stages may interrupt or reduce the efficacy of the processes

\section{AFFILIATIONS}

*Kosair Children's Hospital Research Institute and Division of Paediatric Sleep, Dept of Paediatrics, Medicine, University of Louisville, Louisville, KY, USA, and

\#Dept of Cardiology, Academic Medical Centre, University of Utrecht, Utrecht, The Netherlands.

\section{CORRESPONDENCE}

L. Kheirandish-Gozal Section of Paediatric Sleep Medicine, Dept of Paediatrics, Pritzker School of Medicine University of Chicago 5814 S Maryland Avenue C-113

Chicago IL 60637 USA

E-mail: Igozal@

peds.bsd.uchicago.edu

Received:

July 192009

Accepted after revision:

Nov 272009

First published online: Jan 142010 
underlying memory consolidation. In addition, sleep has been shown to strengthen memories and make them more resistant to interference in both adults [29] and children [30]. Indeed, several studies have now shown that retention of word pairs was significantly increased after sleep, and that sleep enhanced memory performance for faces in both adults and children [3134]. Similarly, non-disrupted sleep leads to improved performance in memory recall, and enhancement of memory performance is only seen after a good night of sleep [29, 30, $35,36]$.

Based on aforementioned considerations, it would be anticipated that intermittent hypoxia and sleep fragmentation would negatively influence academic achievement in children $[37,38]$, even if substantial variability across studies has been observed [39]. Supportive of this assumption is the fact that academic performance and cognitive deficits will improve after treatment of OSA [38]. Nevertheless, it should be further emphasised that at any given level of severity, not all patients with OSA will actually demonstrate the presence of morbid consequences [23]. Therefore, a triple-risk model has been proposed which involves complex interplay between disease severity, environmental conditions and gene-related polymorphisms [6]. We are unaware of any published studies that have specifically assessed whether the process of memory consolidation is altered among children with OSA. Therefore, we hypothesised that children who are referred for symptoms of OSA are more likely than children without OSA to display impairments in the acquisition, consolidation or retrieval of memories.

\section{SUBJECTS AND METHODS}

The study was approved by the University of Louisville Human Research Committee (Louisville, KY, USA). Informed consent was obtained from the legal caregiver of each participant, with assent being obtained from children aged $>7$ yrs. Children aged 6-16 yrs, who were referred for clinical evaluation of habitual snoring and who were scheduled for an overnight polysomnogram (NPSG) at the University of Louisville Paediatric Sleep Medicine Center between October and December 2008, were prospectively approached and invited to participate in a 15 min memory test $\sim 30$ min before the start of their scheduled NPSG. The children were then asked to repeat the memory recall test component immediately after completion of the sleep study the following morning.

Participants were excluded if they suffered from any chronic medical or psychiatric condition, had a genetic syndrome that was known to affect cognitive abilities, or were receiving medications that are known to interfere with memory or sleep onset. Healthy non-snoring children were recruited from an ongoing parallel research study as controls, and were matched for age, sex, ethnicity and level of maternal education.

In addition to standard anthropometric and demographic data, information on prior or current use of medication, presence of comorbidities, history of tympanostomy tube placement and prior surgical removal of adenoids and tonsils was collected. Body mass index (BMI) was calculated from measured height and weight and the BMI z-score was computed using Centers for Disease Control and Prevention growth standards [40] and online software (www.cdc.gov/epiinfo). A BMI z-score $>1.65$ was considered as fulfilling obese criteria.

\section{Memory recall test}

The subjects were shown into their designated private sleep room in the laboratory and were asked to sit comfortably on the bed with the television turned off. The investigator conducting the memory recall test was blinded as to why the sleep study was being performed and was also unaware of the polysomnographic findings. This investigator (M.R. de Jong) was positioned on a chair next to the bed in close proximity and facing the subjects. The parent/guardian was asked not to interfere or help the subjects during testing. Before stating the protocol, subjects were reminded simply about how the test was going to be performed and allowed to ask any questions. The children were then shown a series of 26 colourful animal pictures, all of which were highly familiar to children (e.g. dog, cat, chicken, lion, elephant, giraffe, horse, cow, camel, fish, butterfly, etc.). The child initially identified the animal and then the investigator also named each animal as further corroboration of the adequate recognition of the animal in each picture, while pointing them out page after page. Subjects were allowed $10 \mathrm{~s}$ to look at each animal picture. The book was then closed and the subjects were given 2 min to freely recall any of the animals they could remember without looking at the pictures (fig. 1a). One point was awarded for every correct answer, points were not subtracted for wrong answers and subjects were told that they were allowed to repeat the animal names if they wished to do so.

After the first trial the subjects were allowed to look at the pictures again and go over the animals names. This process was repeated a total of four times in the evening (acquisition phase), followed by a first recall test $10 \mathrm{~min}$ after completion of the fourth trial. During this 10-min interval the child was allowed to watch TV. The morning after the sleep study, within 10-15 min of awakening, the subjects were asked to recall the pictures that they remembered from the previous evening's trials, and the morning score was calculated (fig. 1a).

\section{NSPG assessment}

A standard multichannel NPSG evaluation was performed as described previously [41]. Briefly, the children were studied for $\leqslant 10 \mathrm{~h}$ in a quiet, darkened room in company of a parent or guardian. All children were in bed between 21:00 $\mathrm{h}$ and 22:00 $\mathrm{h}$ and were woken up between 06:00 $\mathrm{h}$ and 07:00 h. No drugs were used to induce sleep. The following parameters were monitored using a computerised system: bilateral electrooculogram; eight channels of electroencephalogram; submental and bilateral anterior tibial electromyograms; and analogue output from a body position sensor (Braebon Medical Corporation, Ogdensburg, NY, USA). The following were also recorded: respiratory effort by chest and abdominal wall movement via respiratory inductive plethysmography; heart rate by electrocardiogram; and airflow, which was triple monitored with a side stream end-tidal capnograph, which also provided breath-by-breath assessment of end-tidal carbon dioxide levels (Welch Allyn OEM Technologies, Beaverton, OR, USA), a nasal pressure cannula and an oronasal thermistor. Arterial oxygen saturation measured by pulse oximetry $\left(\mathrm{Sp}_{\mathrm{p}} \mathrm{O}_{2}\right)$ (Nellcor N-100; Nellcor Inc., Pleasanton, CA, USA) 

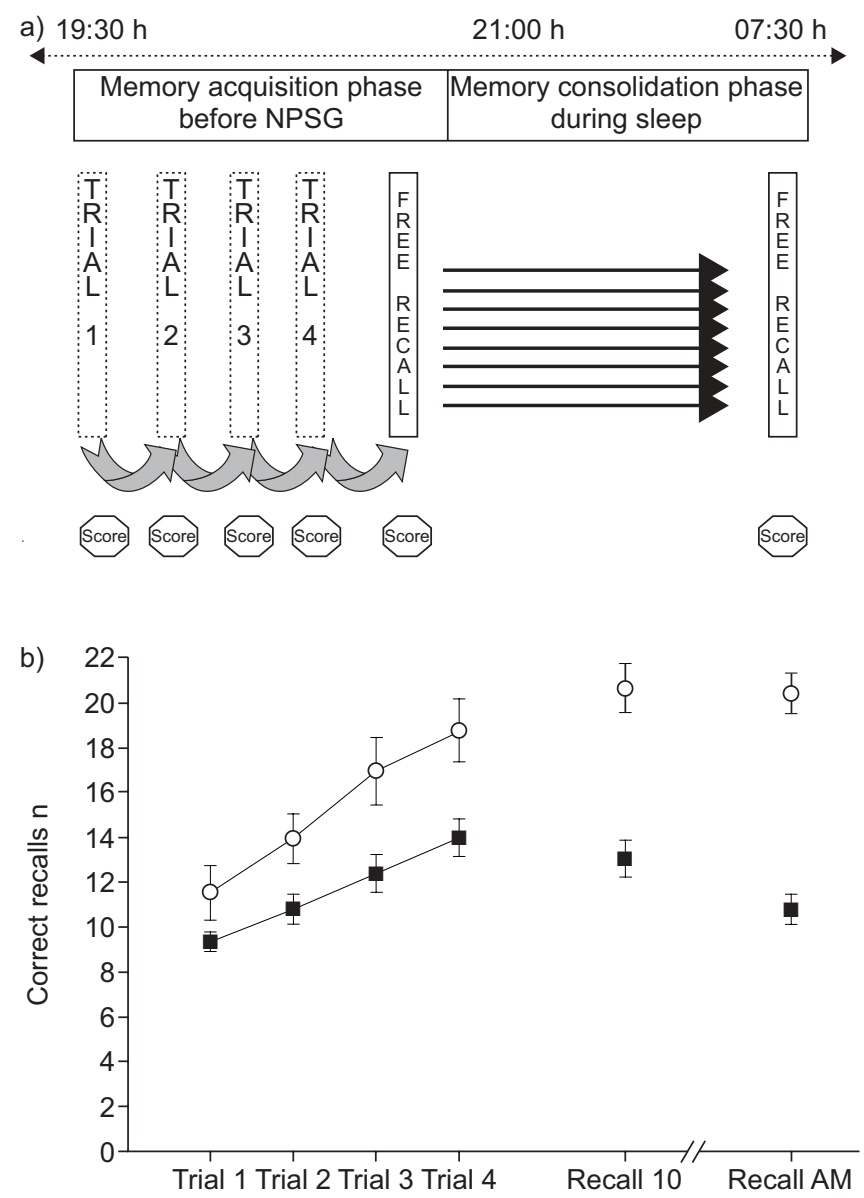

FIGURE 1. a) Schematic diagram of the experimental protocol. NPSG: overnight polysomnogram. b) Visual task learning trajectory performance and immediate and overnight recall performances in 54 children with OSA ( $\mathbf{\square})$ and 17 matched controls $(O)$. Recall 10: recall test 10 min after the fourth trial; Recall AM: recall test performed the morning after the sleep study.

with simultaneous recording of the pulse waveform. All measures were digitised using a commercially available polysomnography system (Stellate Systems, Montreal, Canada). Tracheal sound was monitored with a microphone sensor (Sleepmate Technologies, Midlothian, VA, USA), and a digital time-synchronised video recording was performed.

Sleep architecture and respiratory and arousal events were assessed by standard techniques by an experienced technician using the recent American Academy of Sleep Medicine Guidelines [41, 42]. Central, obstructive and mixed apnoeic events were counted. Obstructive apnoea was defined as the absence of airflow with continued chest wall and abdominal movement for at least two breaths. Hypopnoeas were defined as a decrease in oronasal flow of $\geqslant 50 \%$ with a corresponding decrease in $\mathrm{Sp}_{\mathrm{p}} \mathrm{O}_{2}$ of $\geqslant 4 \%$ and/or arousal. The obstructive apnoea/hypopnoea index (AHI) was defined as the number of apnoeas and hypopnoeas per hour of total sleeping time (TST). The diagnostic criteria for OSA in this study were an obstructive AHI $\geqslant 2$ events $\cdot \mathrm{h}^{-1}$ TST in the presence of snoring during NPSG. Control children were defined as non-snoring children with an obstructive AHI $<1$ events $\cdot \mathrm{h}^{-1}$ TST [43].
Arousals were expressed as the total number of arousals per hour of sleep time (arousal index), and included respiratoryrelated (occurring immediately following an apnoea, hypopnoea or snore), technician-induced and spontaneous arousals. In addition, awakening episodes after sleep onset were recorded and counted.

\section{Data analysis}

Data are presented as mean \pm SE unless otherwise stated. Categorical data were compared using the Chi-squared test followed by Fisher Exact corrections. The individual score for each trial from the evening session was calculated and the average evening and morning scores were derived. Comparisons across the evening and morning and across the two groups (OSA and controls) were initially performed using two-way ANOVA for repeated measures followed by post hoc tests and Bonferroni correction for multiple comparisons. Subsequently, data were submitted to a temporal principal components analysis (PCA) using a covariance matrix and varimax rotation. The PCA identified intervals of high variability in individual recall performances during the trial (i.e. factors). The rotated factor scores served as dependent variables in a repeated measure ANOVA that was used to identify the possible causes of the variability. The analysis design included the performance during recall trial (learning phase, immediate, morning) $\times$ sleep variable $(\mathrm{AHI}$, arousal index, respiratory arousal index, nadir $\mathrm{Sp}_{\mathrm{p}} \mathrm{O}_{2}$, maximal endtidal $\left.\mathrm{CO}_{2}\right) \times$ group (control, OSA) $\times$ age $\times$ BMI variables The Greenhouse-Geisser correction was applied where appropriate. A p-value $<0.05$ was considered to be statistically significant.

\section{RESULTS}

In total, 71 subjects were recruited, with 54 subjects in the OSA group and 17 subjects in the control group. The mean age was $\sim 11$ yrs (table 1). Age, sex, ethnicity and level of maternal education were similar in both groups (table 1). However, OSA children had significantly higher BMI z-scores $(p<0.01)$, and the proportion of obese children was also significantly larger $(p<0.03)$. The OSA children had a significantly higher AHI $\left(6.3 \pm 1.5\right.$ events $\cdot h^{-1} \quad$ TST; $\left.p<0.0001\right)$ than the controls $\left(0.6 \pm 0.1\right.$ events $\cdot h^{-1}$ TST). The proportion of subjects in the control group and OSA group with a history of seasonal allergies was similar $(p=N S)$. Similarly, the frequency of reporting physician-diagnosed asthma did not differ among the two groups $(p=N S)$.

Comparisons of free recall performance across the learning trials revealed significant differences between the OSA children and the controls (fig. 1), with OSA children showing small, albeit significantly lower, performances after the first trial $(\mathrm{p}<0.05)$ and markedly slower improvements in trial recall over the second to fourth consecutive trials (fig. 1; $p<0.001$ ), finally reaching lower final performance scores at the end of the four trials (fig. $1 ; p<0.0001$ ), as well as during the immediate $10-\mathrm{min}$ recall $(\mathrm{p}<0.0001)$. Morning recall scores were not only significantly lower among OSA children compared to controls $(\mathrm{p}<0.001)$, but were also lower compared to their corresponding evening performance $(p<0.001)$. In contrast, there were no evening-morning differences in the control group ( $\mathrm{p}<0.01$ versus OSA, two-way ANOVA) (fig. 1). 


\begin{tabular}{|c|c|c|c|}
\hline \multirow[t]{2}{*}{ TABLE 1} & \multirow[b]{2}{*}{ Control } & $\begin{array}{l}\text { group ar } \\
\text { SSA) group }\end{array}$ & \multirow[b]{2}{*}{ p-value } \\
\hline & & OSA & \\
\hline Subjects $n$ & 17 & 54 & \\
\hline Age yrs & $12.1 \pm 0.9$ & $10.5 \pm 0.5$ & NS \\
\hline Sex male/female $n$ & $8 / 9$ & $25 / 29$ & NS \\
\hline Ethnicity C/AA n & $13 / 4$ & $41 / 13$ & NS \\
\hline Maternal Education & $5 \mathrm{HS} / 12 \mathrm{C}$ & $14 \mathrm{HS} / 40 \mathrm{C}$ & NS \\
\hline BMI z-score & $0.92 \pm 0.16$ & $1.48 \pm 0.21$ & $<0.01$ \\
\hline Seasonal allergies & 52 & 56 & NS \\
\hline Asthma & 23 & 32 & NS \\
\hline Sleep onset latency $\mathrm{min}$ & $35.7 \pm 6.8$ & $24.6 \pm 4.1$ & $<0.04$ \\
\hline TST $\min$ & $441.9 \pm 8.8$ & $448.8 \pm 8.8$ & NS \\
\hline Non-REM sleep $1 \%$ TST & $6.2 \pm 0.9$ & $8.0 \pm 0.6$ & NS \\
\hline Non-REM sleep 2 \% TST & $43.1 \pm 2.9$ & $50.3 \pm 1.9$ & $<0.05$ \\
\hline Non-REM sleep 3-4 \% TST & $28.5 \pm 2.2$ & $22.5 \pm 1.6$ & $<0.05$ \\
\hline REM sleep \% TST & $22.2 \pm 1.9$ & $19.2 \pm 1.2$ & $<0.05$ \\
\hline Sleep efficiency \% & $86.4 \pm 1.7$ & $85.6 \pm 1.3$ & NS \\
\hline AHI events $\cdot h^{-1}$ TST & $0.6 \pm 0.1$ & $6.3 \pm 1.1$ & $<0.0001$ \\
\hline Nadir $\mathrm{Sp}, \mathrm{O}_{2} \%$ & $94.5 \pm 0.3$ & $87.5 \pm 1.3$ & $<0.0001$ \\
\hline TAl events $\cdot h^{-1}$ TST & $8.6 \pm 1.2$ & $11.9 \pm 1.7$ & $<0.01$ \\
\hline RAl events $\cdot h^{-1}$ TST & $0.4 \pm 0.1$ & $2.9 \pm 0.4$ & $<0.0001$ \\
\hline PET,CO ${ }_{2}$,peak $\mathrm{mmHg}$ & $47.9 \pm 0.6$ & $52.5 \pm 0.7$ & $<0.002$ \\
\hline
\end{tabular}

Data are presented as mean \pm SE or $\%$, unless otherwise stated. C: white Caucasian; AA: African-American; BMI: body mass index; TST: total sleep time; REM: rapid eye movement; $A H I$ : apnoea/hypopnoea index; $S p, \mathrm{O}_{2}$ : arterial oxygen saturation measured by pulse oximetry; TAl: total arousal index; RAl: respiratory arousal index; $P \mathrm{ET}, \mathrm{CO}_{2}$,peak: peak end-tidal carbon dioxide tension HS: hispanic; NS: nonsignificant.

The PCA identified four sleep-related factors that accounted for $52.2 \%$ of the total variance in the learning trajectory, namely AHI $(5.4 \%)$, respiratory arousal index $(37.8 \%)$, REM sleep expressed (4.7\% TST) and wake time after sleep onset events (5.3\%). ANOVA further revealed that the respiratory arousal index remained a significant predictor of both learning slope and morning recall function, even after adjusting for age, sex, ethnicity, maximal individual performance and total sleep duration as potential confounders $(p<0.001)$.

\section{DISCUSSION}

This study shows reduced acquisition and recall of animal picture-based learning and retention in children with OSA compared to matched control subjects. Our findings are in agreement with the study by KAEMINGK et al. in children [11] and SALORIO et al. in adults [44], who found differences in the pattern of acquisition and recall of new information in patients with OSA when compared to controls. Although a definitive causal relationship between SDB and declarative memory deficits can only be established by a randomised interventional study, the present findings add further support to the hypothesis that OSA adversely influences aspects of dynamic memory acquisition and performance in children.

The decreased recall performance found in the OSA group could be mediated, at least in part, by the underlying sleep fragmentation and disruption of sleep integrity and continuity that clearly accompany the presence of OSA. While the severity of hypoxia and the presence of recurrent hypoxic events have been associated with some of the deficits in executive function (which facilitates learning and long-term memory) [22], sleep fragmentation and restriction seem to preferentially account for the variance in attention. Thus, sleep disturbance and fragmentation could elicit attention deficits and failure to use an efficient learning strategy, or be associated with the inability to appropriately process novel information. It has been suggested that children with OSA have problems with sustaining attention rather than with short-term attention [45]. Decreased concentration may explain some of the differences in memory performance found in this study. According to the findings reported by SALORIO et al. [44], reduced memory performance could be accounted for by problems of encoding, rather than by deficits in the consolidation of new memories in individuals with OSA. The authors also showed that the use of executive strategies (semantic clustering) was reduced in children with OSA [44].

Of note, we did not specifically evaluate children for the presence of attention deficit hyperactivity disorder (ADHD). Although none of the children enrolled were receiving psychostimulants or reported this diagnosis in their medical history, we can not exclude the presence of ADHD with certainty. It has now been repeatedly reported that problems regarding attention and behaviour pattern resembling ADHD are often encountered among children suffering from OSA [17, 18]. A significant relationship between the degree of sleep disturbance and the severity of OSA in relation to the magnitude of behavioural changes has also been observed. Thus, the potential inclusion of such children could have influenced the current findings, particularly since children with ADHD are known to perform less well in memory studies $[13,15]$. However, it should be emphasised that, as reported by HALBOWER et al. [46], when children with ADHD were excluded from the OSA group analysis, the strength of the association between OSA and cognitive tasks did not change, suggesting that the inclusion of children with ADHD may not have affected our results after all.

The study was designed as a case-control approach, whereby OSA subjects and controls were carefully matched for several demographic characteristics. However, the wide range of age among the participants could have influenced the results, since the manifestations of OSA vary by age and a decline in the severity of OSA may be seen from the age of 9 yrs onwards [46]. In other words, this expanded age range may have reduced the magnitude of the effect of OSA on the task performance studied herein [47]. Again, the findings of KURNATOWSKI et al. [47] did not support such contention, since in their study they found that both children aged 6-9 yrs and 10-13 yrs had problems with memory and sustaining attention to the same degree. Although the groups were matched for age, sex and ethnicity, we did not match for other potential confounders such as educational level, intelligence and socioeconomic background. It has been shown that children with higher intelligence may not be as likely to manifest the detrimental effect of OSA on cognition since, although they might score lower than their original potential, their performances may still fall within "normal" limits [48]. Thus, the lack of insight into actual learning potential constitutes a 
formidable problem in the evaluation of studies involved in the assessment of intelligence and memory. In addition, KAEMINGK et al. [11] have shown that a higher socioeconomic status may protect against the influences of OSA on cognitive function. We should also point out that the OSA children were recruited from a referral and symptomatic, habitually snoring population rather than from a general community-based, habitually snoring population and, therefore, this referral cohort could be disproportionately represented with subjects with enhanced end-organ susceptibility to the OSA disease process. Finally, in this exploratory study, subjects were not matched for BMI z-score, such that a higher proportion of subjects in the OSA group were obese. Considering the role of obesity as a major risk factor for OSA $[7,8]$, and the fact that obesity per se is intrinsically associated with an increased risk for cognitive deficits [48], the respective contributions of OSA and obesity remain hitherto undefined, and will have to await an intervention study.

Regarding the potential contribution of specific sleep-related variables to our findings, KAEMINGK et al. [11] have previously indicated that the arousal index may influence memory performance, and that the percentage of stage 1 sleep (in the AHI $\geqslant 5$ events $\cdot h^{-1}$ TST group) emerged as negatively associated with overall learning across trials, as well as with immediate and delayed recall. Our current results add further novel insights to the putative contributions by respiratory arousal index and, to a lesser extent, by other alterations in sleep integrity to the overall performance during our visually based task. However, although our test assessed global episodic memory and measured free recall and delayed recall, its internal validity has not been firmly established. In addition, our experimental testing paradigm only considered one aspect of memory which may not be the most sensitive test that could be implemented, since vulnerability could be both task-dependent and individual-dependent.

Notwithstanding all aforementioned considerations, the significant findings clearly support an effect of OSA on the memory functions that were assessed using this learning and testing paradigms. We should also point out that an important and advantageous methodological issue in the fact that all of the tests were performed by the same investigator and, as such, operator-introduced variance was thereby reduced.

In summary, differences in the acquisition of a pictorial task in children with OSA are apparent when compared to control subjects. Although the subjects with OSA are ultimately capable to achieve learning performance after four trials, the acquisition trajectory is substantially different and slower in OSA, and may require many additional trials to reach the performance levels achieved by controls. Furthermore, recall is also affected both immediately and during the following day. These deficits appear to be mainly accounted for by morbid outcomes originating from both the respiratory and sleep disturbance, with a more prominent effect appearing to be dictated by the magnitude of sleep fragmentation. In the contextual setting of our experimental approach, we can not conclude at this time whether OSA children have: 1) difficulty memorising new information due to an impaired ability to form adequate learning strategies; 2) suffer from impaired encoding; 3) suffer from altered retrieval; or 4) a combination of the aforementioned. Furthermore, the reversibility of such altered memory formation processes will have to await an intervention study. However, this study has enabled the development of a simple test that can be easily incorporated into the routine administration of an overnight sleep study in the process of evaluating habitually snoring children. Such an approach may potentially provide important additional information that may assist in the process of clinical management decisions regarding type of treatment (i.e. adenotonsillectomy versus anti-inflammatory therapy) and the urgency and priority for treatment of OSA in children by helping identify those children who are more vulnerable and who should, therefore, receive earlier therapeutic intervention.

\section{SUPPORT STATEMENT}

The research was supported by a National Institute of Health grant (HL-65270).

\section{STATEMENT OF INTEREST}

Statements of interest for L. Kheirandish-Gozal and D. Gozal can be found at www.erj.ersjournals.com $/ \mathrm{misc} /$ statements.dtl

\section{REFERENCES}

1 Standards and indications for cardiopulmonary sleep studies in children. American Thoracic Society. Am J Respir Crit Care Med 1996; 153: 866-878.

2 Muzumdar H, Arens R. Diagnostic issues in pediatric obstructive sleep apnea. Proc Am Thorac Soc 2008; 5: 263-273.

3 Kheirandish-Gozal L, Gozal D. The multiple challenges of obstructive sleep apnea in children: diagnosis. Curr Opin Pediatr 2008; 20: 650-653.

4 Lumeng JC, Chervin RD. Epidemiology of pediatric obstructive sleep apnea. Proc Am Thorac Soc 2008; 5: 242-252.

5 Section on Pediatric Pulmonology, Subcommittee on Obstructive Sleep Apnoea Syndrome, American Academy of Pediatrics, Clinical practice guideline: diagnosis and management of childhood obstructive sleep apnea syndrome. Pediatrics 2002; 109: 704-712.

6 Gozal D, Kheirandish L. Oxidant stress and inflammation in the snoring child: confluent pathways to upper airway pathogenesis and end-organ morbidity. Sleep Med Rev 2006; 10: 83-96.

7 Arens R, Marcus CL. Pathophysiology of upper airway obstruction: a developmental perspective. Sleep 2004; 27: 997-1019.

8 Dayyat E, Kheirandish-Gozal L, Sans Capdevila O, et al. Obstructive sleep apnea in children: relative contributions of body mass index and adenotonsillar hypertrophy. Chest 2009; 136: 137-144.

9 Gozal D, Pope DW. Snoring during early childhood and academic performance at ages thirteen to fourteen years. Pediatrics 2001; 107: 1394-1399.

10 Gozal D, Kheirandish-Gozal L. The multiple challenges of obstructive sleep apnea in children: morbidity and treatment. Curr Opin Pediatr 2008; 20: 654-658.

11 Kaemingk KL, Pasvogel AE, Goodwin JL, et al. Learning in children and sleep disordered breathing: findings of the Tuscon Children's Assessment of Sleep Apnea (TuCASA) prospective cohort study. J Int Neuropsychol Soc 2003; 9: 1016-1026.

12 Kheirandish L, Gozal D, Pequignot J-M, et al. Intermittent hypoxia during development induces long-term alterations in spatial working memory, monoamines, and dendritic branching in rat frontal cortex. Pedriatr Res 2005; 58: 594-599.

13 Blunden S, Lushington K, Kennedy D, et al. Behavior and neurocognitive performance in children aged 5-10 years who 
snore compared to controls. J Clin Exp Neuropsychol 2000; 22: 554-568.

14 Ellenbogen JM. Cognitive benefits of sleep and their loss due to sleep deprivation. Neurology 2005; 64: E25-E27.

15 Gottlieb DJ, Chase C, Vezina RM, et al. Sleep-disordered breathing symptoms are associated with poorer cognitive function in 5-yearold children. J Pediatr 2004; 145: 458-464.

16 Kheirandish L, Gozal D. Neurocognitive dysfunction in children with sleep disorders. Dev Sci 2006; 9: 388-399.

17 O'Brien LM, Holbrook CR, Mervis CB, et al. Sleep and neurobehavioral characteristics of 5- to 7-year-old children with parentally reported symptoms of attention-deficit/hyperactivity disorder. Pediatrics 2003; 111: 554-563.

18 O'Brien LM, Mervis CB, Holbrook CR, et al. Neurobehavioral correlates of sleep-disordered breathing in children. I Sleep Res 2004; 13: 165-172.

19 Rhodes SK, Shimoda KC, Waid LR, et al. Neurocognitive deficits in morbidly obese children with obstructive sleep apnea. J Pediatr 1995; 127: 741-744.

20 Montgomery-Downs HE, Gozal D. Snore-associated fragmentation in infancy: mental development effects and contribution of secondhand cigarette smoke exposure. Pediatrics 2006; 117: 496-502.

21 Montgomery-Downs HE, Gozal D. Toddler behavior following polysomnography: effects of unintended sleep disturbance. Sleep 2006; 29: 1282-1287.

22 Beebe DW, Gozal D. Obstructive sleep apnea and the prefrontal cortex: towards a comprehensive model linking nocturnal upper airway obstruction to daytime cognitive and behavioral deficits. J Sleep Res 2002; 11: 1-16.

23 Gozal D, Crabtree VM, Capdevila OS, et al. C-reactive protein, obstructive sleep apnea, and cognitive dysfunction in school-aged children. Am J Respir Crit Care Med 2007; 176: 188-193.

24 Garetz SL, Arbor A. Behavior, cognition, and quality of life after adenotonsillectomy for pediatric sleep-disordered breathing: summary of the literature. Otolaryngol Head Neck Surg 2008; 138: Suppl. 1, S19-S26.

25 Sans Capdevila O, Crabtree VM, Kheirandish-Gozal L, et al. Increased morning brain natriuretic peptide levels in children with nocturnal enuresis and sleep disordered breathing: a communitybased study. Pediatrics 2008; 121: e1208-e1214.

26 Baldassari CM, Mitchell RB, Schubert C, et al. Pediatric obstructive sleep apnea and quality of life: a meta-analysis. Otolaryngol Head Neck Surg 2008; 138: 265-273.

27 Tarasiuk A, Simon T, Tal A, et al. Adenotonsillectomy in children with obstructive sleep apnea syndrome reduces healthcare utilization. Pediatrics 2004; 113: 351-356.

28 Stickgold R. Sleep-dependent memory consolidation. Nature 2005; 437: 1272-1278.

29 Ellenbogen JM, Hulbert JC, Stickgold R, et al. Interfering with theories of sleep and memory: sleep, declarative memory, and associative interference. Curr Biol 2006; 16: 1290-1294.

30 Hill CM, Hogan AM, Karmiloff-Smith A. To sleep, perchance to enrich learning? Arch Dis Child 2007; 92: 637-643.
31 Stickgold R, Walker MP. Sleep-dependent memory consolidation and reconsolidation. Sleep Med 2007; 8: 331-343.

32 Walker MP, Stickgold R. Sleep, memory, and plasticity. Annu Rev Psychol 2006; 57: 139-166.

33 Backhaus J, Hoeckesfeld R, Born J, et al. Immediate as well as delayed post learning sleep but wakefulness enhances declarative memory consolidation in children. Neurobiol Learn Mem 2008; 89: 76-80.

34 Wagner U, Kashyap N, Diekelmann S, et al. The impact of postlearning sleep vs wakefulness on recognition memory for faces with different facial expressions. Neurobiol Learn Mem 2007; 87: 679-687.

35 Gais S, Born J. Declarative memory consolidation: mechanisms acting during human sleep. Learn Mem 2004; 11: 679-685.

36 Ellenbogen JM, Payne JD, Stickgold R. The role of sleep in declarative memory consolidation: passive, permissive, active or none? Curr Opin Neurobiol 2006; 16: 716-722.

37 Gozal D, Kheirandish-Gozal L. Neurocognitive and behavioral morbidity in children with sleep disorders. Curr Opin Pulm Med 2007; 13: 505-509.

38 Gozal D. Sleep-disordered breathing and school performance in children. Pediatrics 1998; 102: 616-620.

39 Blunden SL, Beebe DW. The contribution of intermittent hypoxia, sleep debt and sleep disruption to daytime performance deficits in children: consideration of respiratory and non-respiratory sleep disorders. Sleep Med Rev 2006; 10: 109-118.

40 Centers for Disease Control and Prevention. CDC Growth Charts. www.cdc.gov/growthcharts

41 Redline S, Budhiraja R, Kapur V, et al. The scoring of respiratory events in sleep: reliability and validity. J Clin Sleep Med 2007; 3: $169-200$

42 Grigg-Damberger M, Gozal D, Marcus CL, et al. The visual scoring of sleep and arousal in infants and children. J Clin Sleep Med 2007; 3: 201-240.

43 Montgomery-Downs HE, O'Brien LM, Gulliver TE, et al. Polysomnographic characteristics in normal preschool and early school-aged children. Pediatrics 2006; 117: 741-753.

44 Salorio CF, White DA, Piccirillo J, et al. Learning, memory, and executive control in individuals with obstructive sleep apnea syndrome. J Clin Exp Neuropsychol 2002; 24: 93-100.

45 Beebe DW. Neurobehavioral morbidity associated with disordered breathing during sleep in children: a comprehensive review. Sleep 2006; 29: 1115-1134.

46 Halbower AC, Degaonkar M, Barker PB, et al. Childhood obstructive sleep apnea associates with neuropsychological deficits and neuronal brain injury. PLoS Medicine 2006; 3: 1391-1402.

47 Kurnatowski P, Putynski L, Lapienis M, et al. Neurocognitive abilities in children with adenotonsillar hypertrophy. Int J Pediatr Otorhinolaryngol 2006; 70: 419-424.

48 Sans Capdevilla O, Kheirandish-Gozal L, Dayyat E, et al. Pediatric obstructive sleep apnea: complications, management and longterm outcomes. Proc Am Thorac Soc 2008; 5: 274-282. 